Brit. Heart f., 1967, 29, 859.

\title{
Changes in Cardiac Output and Stroke Volume During First Four Months After Cardiac Infarction
}

\author{
FRANK NAGER ${ }^{\star}$, MICHAEL THOMAS, AND JOHN SHILLINGFORD \\ From the Medical Research Council's Cardiovascular Research Unit, \\ Royal Postgraduate Medical School, London W.12
}

The circulatory changes that occur within the first four months after cardiac infarction have not been studied to the same extent as those occurring during the acute phase of the illness. Measurements of cardiac output and stroke volume have been made in some patients before leaving hospital (Pritchard and Hellerstein, 1950; Gammill et al., 1955; Lee, 1957; Broch et al., 1959; Thomas, Malmcrona, and Shillingford, 1965b), but in comparatively few patients have measurements been made over a longer period of two months (Smith, Wikler, and Fox, 1954; Murphy et al., 1963). In a single report results of investigations made up to three to five months after the acute illness are given (Malmcrona and Varnauskas, 1964).

The purpose of this study was to define more completely the changes in cardiac output and stroke volume that may occur during the first four months after cardiac infarction. The conclusions are based on 126 hæmodynamic investigations made in conjunction with clinical observations in 22 patients. At each investigation the circulatory response to a standard postural change (Thomas and Shillingford, 1965) was studied.

\section{Patients and Methods}

Studies were made of 22 patients, 18 men and 4 women, whose ages ranged from 32 to 80 years, mean 57 years. Five other patients in whom the follow-up studies were not completed owing to their death in hospital or during the early convalescent period have been excluded. All patients had a clinical history and electrocardiographic signs typical of myocardial infarction. The serum lactic dehydrogenase was raised in $\mathbf{1 7}$

Received May 1, 1967.

* Present address: Kantonsspital Zürich, Medizinische Universitätsklinik, Zürich, Switzerland. patients. Four patients $(8,9,11,15)$ had had a myocardial infarction in the past. Four $(5,13,16,22)$ were known to have had systemic hypertension before myocardial infarction. Hypertension of varying degree became apparent during the follow-up period in six patients $(9,15,17,18,19,20)$. One (19) suffered from mild diabetes mellitus and three $(1,5,11)$ had evidence of chronic bronchitis.

During the follow-up period three patients $(7,11,13)$ were treated with digoxin and diuretics; one hypertensive patient (13) received bethanidine; another hypertensive patient (22) received chlorothiazide before the last investigation; and one (16) had a probable further myocardial infarction before the last investigation.

All patients were in sinus rhythm at the time of hæmodynamic study.

All 22 patients were studied serially in the early convalescent period and after discharge from hospital. In addition, 14 of the 22 patients were studied during the acute illness, as detailed. Measurements in the acute phase (first week following infarction) were made in a special intensive care unit with equipment permanently installed (Shillingford and Thomas, 1964). Subsequent measurements during the early convalescent period (second to fifth week) were conducted in a catheter room and were in general made during the second or third week, as detailed. After discharge from hospital, patients were studied during the periods 4-5 weeks, 6-8 weeks, 9-13 weeks, and 15-22 weeks, with a few exceptions as noted.

Each hæmodynamic study consisted of measurements of heart rate, cardiac output, and arterial blood pressure. Cardiac output was measured by a dye dilution technique (Gabe and Shillingford, 1961 ; Thomas, Malmcrona, and Shillingford, 1965a), using the photoelectric earpiece (Cambridge Instrument Co.) and Coomassie Blue dye (I.C.I.). A known quantity of dye (approx. $50 \mathrm{mg}$.) was injected from a special syringe (Gabe and Shillingford, 1961) through a fine polyethylene catheter (PE 60 Intramedic U.S.A.) inserted percutaneously by Seldinger technique and advanced to the great veins. The first dye 859 
extracted from the plasma of a blood sample taken three minutes after injection. Dye concentration was measured by spectrophotometry. Subsequent relative cardiac outputs were calculated from the reciprocal of the areas of the extrapolated dye curves.

Heart rate was derived from the dye curve by using slightly incomplete pulse rejection on the dye recorder. Arterial pressure was measured by sphygmomanometry.

In six patients $(5,6,7,8,10,11)$ the cardiac outputs during the acute phase were calculated by the direct Fick principle. Oxygen consumption was measured by collecting expired air in a Douglas bag. The $\mathrm{O}_{2}$ and $\mathrm{CO}_{2}$ tensions of the expired air were determined by use of a polarographic electrode (Instrument Laboratory IL 113) and the volume was measured by passing it through a dry gas meter. Arterial blood was obtained from the brachial artery and mixed venous blood by advancing the catheter into the pulmonary artery. The oxygen content of the arterial and mixed venous blood was calculated from the $\mathrm{Po}_{2}, \mathrm{pH}$, and $\mathrm{Hb}$, using Dill's oxygen dissociation tables.

Patients studied during the acute phase were lying flat. All other investigations in the convalescent period and after discharge from hospital took place with the patients lying on an electrically-driven tipping table. Initial measurements were made in a $20^{\circ}$ feet down position. The procedure undertaken for follow-up studies was to insert the catheter with the patient lying flat. The patient's position was then changed to the $20^{\circ}$ feet down position, and after approximately 5 minutes the first two measurements of cardiac output, heart rate, and blood pressure were made. The patient's position was then changed to the horizontal and the legs raised by means of a wooden support. After 3-4 minutes two further measurements of heart rate, cardiac output, and blood pressure were made.

\section{RESULTS}

Results of the hæmodynamic measurements are summarized in the Table and Fig. 1-6.

\section{Hamodynamic Changes}

(1) Cardiac Output. Measurements of cardiac output in the acute phase (14 patients) ranged from 2.40 to $6.74 \mathrm{l} / \mathrm{min}$; at the beginning of the convalescent period (22 patients) from 2.97 to 6.8 1./min. and at the end of the period of follow-up (22 patients) from $3 \cdot 10$ to $6 \cdot 861 . / \mathrm{min}$.

Acute phase-early convalescence. Of the 14 patients studied in the acute phase, $2(20,22)$ had a cardiac output above $5 \mathrm{l}$./min., $9(5,6,10,14,15$, $16,17,19,21)$ had a cardiac output between 4.0 and $5 \cdot 01$. $/$ min., and $3(7,8,11)$ had a cardiac output less than $4 \mathrm{l}$./min.

Within the period between the acute illness and the first study in the early convalescent period, of the two patients with initial cardiac output above 51 ./min., one (20) showed an increase and the other
(22) a fall. Of the 9 patients with initial cardiac output between $4 \cdot 0$ and $5 \cdot 0$ 1./min., $4(6,10,14,16)$ showed an increase: in patients 10 and 14 this was of the order of $21 . / \mathrm{min}$. Four $(5,15,17,19)$ of these 9 patients showed no change and one (21) showed a fall.

Early convalescence-end of follow-up. All 22 patients were studied serially in the period between early convalescence and the third or fourth month. At the time of the first study in the convalescent period, 12 patients $(2,3,4,6,8,9,10,13,14,16$, $18,20)$ had a cardiac output above $51 . / \mathrm{min}$. Seven patients $(5,7,12,15,17,19,22)$ had a cardiac output between 4.0 and $5.01 \cdot \mathrm{min}$., and $3(1,11,21)$ had a cardiac output of less than $4.0 \mathrm{l} / \mathrm{min}$.

During the period of follow-up from the second week, of the 12 patients with a cardiac output above $5.0 \mathrm{l} / \mathrm{min}$. in early convalescence, only one (9) showed a further rise. One (6) showed a transient increase and then a fall to the initial level. Four patients $(2,3,13,18)$ showed no change; six $(4,8$, $10,14,16,20)$ showed a fall in cardiac output, but in only $3(14,16,20)$ did cardiac output fall below $5 \mathrm{l} / \mathrm{min}$. In one (16) of these three patients a further myocardial infarction at the twelfth week may have contributed and in another (20) increase of blood pressure to $200 / 110 \mathrm{~mm}$. $\mathrm{Hg}$ occurred during the follow-up period.

(2) Stroke Volume. Results of stroke volume measurements are given with reference to three ranges:- those patients with stroke volume above $60 \mathrm{ml}$. (Group 1), those patients with stroke volume between 40 and $60 \mathrm{ml}$. (Group 2), and those patients with stroke volume of less than $40 \mathrm{ml}$. (Group 3). Changes in stroke volume in patients who had values within these ranges at the first study in early convalescence are illustrated in Fig. 1, 2, and 3.

Measurements of stroke volume in the 14 patients studied in the acute phase ranged from 20 to $87 \mathrm{ml}$., in all 22 patients at the beginning of convalescence from 38 to $97 \mathrm{ml}$., and at the end of the period of follow-up from 35 to $105 \mathrm{ml}$.

Acute phase-early convalescence. Measurements of stroke volume in the acute phase in the 14 patients previously detailed, as compared with the first measurement in early convalescence, showed the following changes. Of 3 patients $(14,16,20)$ with a lowest value of stroke volume in the acute illness of above $60 \mathrm{ml}$., one (14) remained of the same order and two $(16,20)$ subsequently showed an increase. Of 9 patients $(5,6,8,10,15,17,19,21$, 22) with stroke volume between 40 and $60 \mathrm{ml}$. in the acute phase, $6(5,6,8,10,15,17)$ showed an increase 


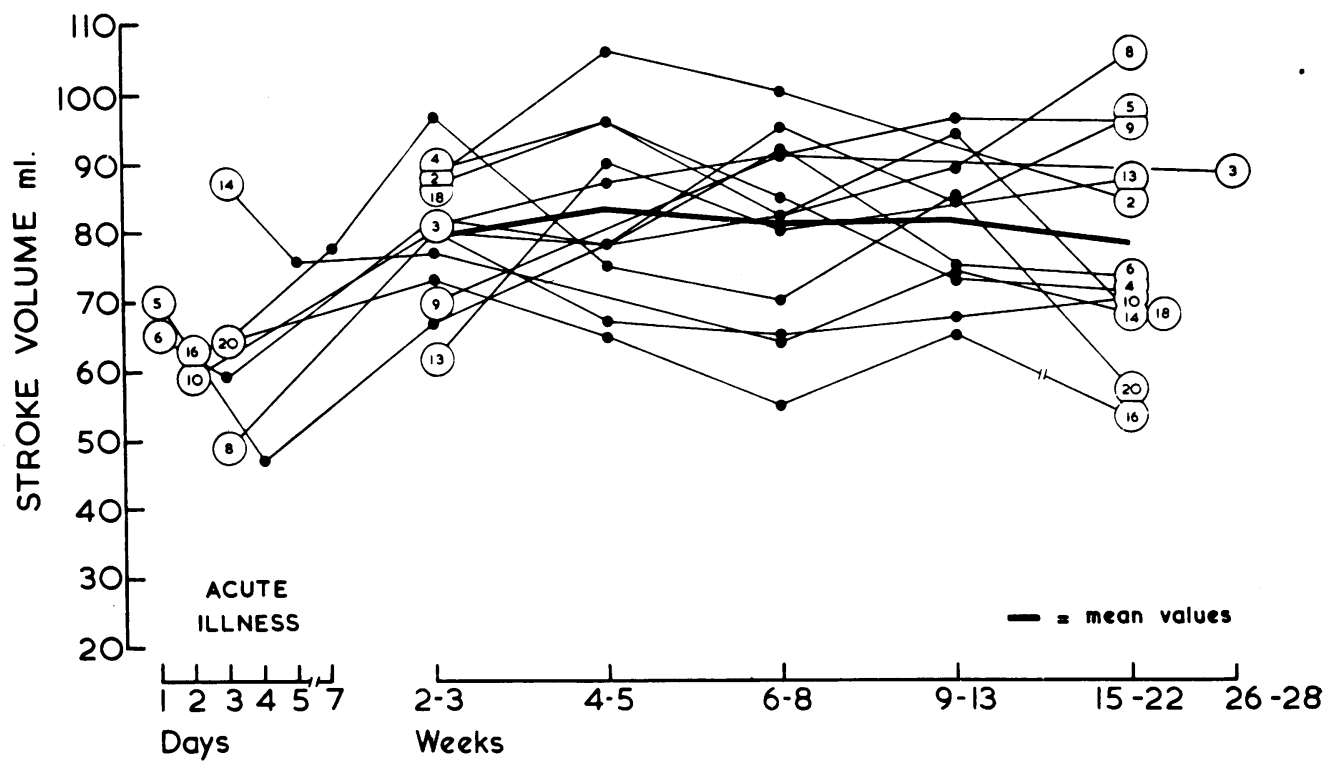

TIME OF INVESTIGATION

FIG. 1.-Changes in stroke volume occurring in those patients with stroke volume above $60 \mathrm{ml}$. at the first study in the early convalescent period (Group I). The thick line represents mean values. Figures in circles are patient numbers.

during the following week, one (21) showed a fall and in the remaining two there was no significant change. Two patients $(7,11)$ had a stroke volume between 20 and $40 \mathrm{ml}$. in the acute phase. In the following week both showed an increase, greater in patient 7 as compared with patient 11 .
Early convalescence-end of follow-up. Thirteen patients $(2,3,4,5,6,8,9,10,13,14,16,18,20)$ had a stroke volume greater than $60 \mathrm{ml}$. at the first investigation in early convalescence. Of these, $4(5,8,9,13)$ showed a further increase, $5(2,3,6$, $10,14)$ showed no change, and $4(4,16,18,20)$

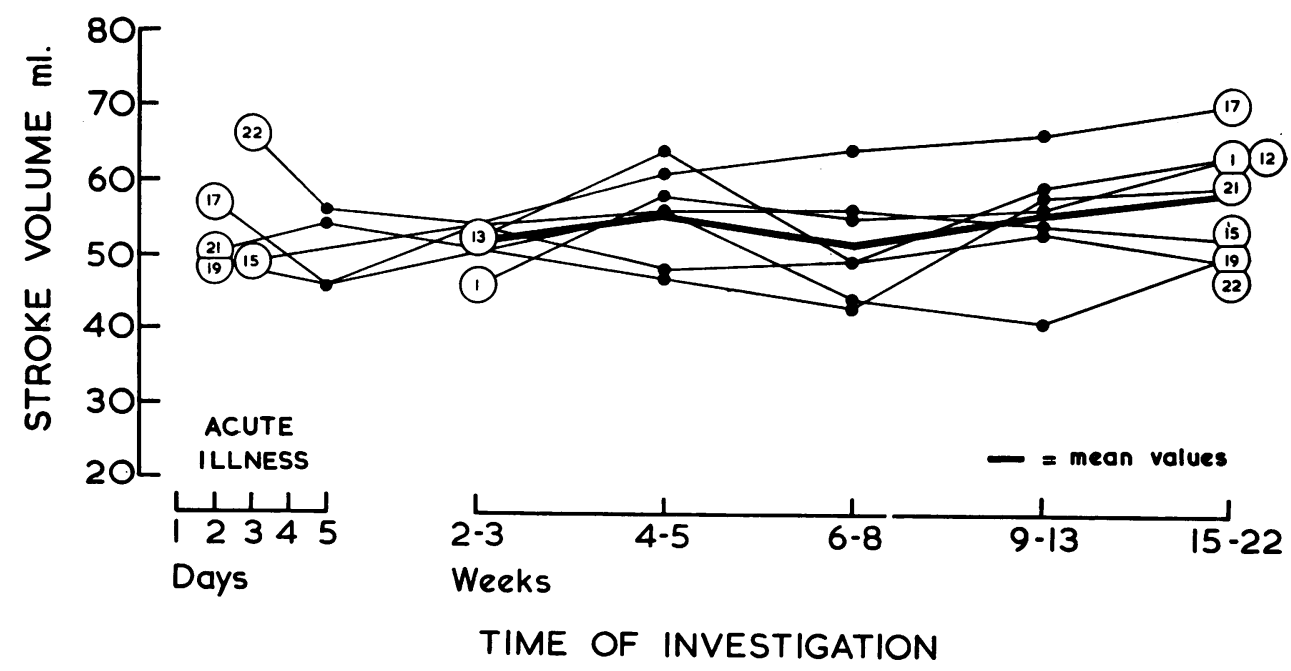

FIG. 2.-Changes in stroke volume occurring in those patients with stroke volume between 40 and $60 \mathrm{ml}$. at the first study in the early convalescent period (Group II). The thick line represents mean values. 
TABLE

HÆMODYNAMIC DATA

\begin{tabular}{|c|c|c|c|c|c|c|c|c|c|}
\hline \multirow[b]{2}{*}{$\begin{array}{c}\text { Patient } \\
\text { No. }\end{array}$} & \multirow[b]{2}{*}{$\begin{array}{c}\text { Days } \\
\text { (D1-D7) or } \\
\text { weeks }(2-28) \\
\text { after } \\
\text { infarction }\end{array}$} & \multicolumn{4}{|c|}{ Position 1} & \multicolumn{4}{|c|}{ Position 2} \\
\hline & & $\begin{array}{c}\text { Heart } \\
\text { rate } \\
\text { (beats/min.) }\end{array}$ & $\begin{array}{l}\text { Cardiac } \\
\text { output } \\
\text { (1./min.) }\end{array}$ & $\begin{array}{c}\text { Stroke } \\
\text { volume } \\
\text { (ml.) }\end{array}$ & $\begin{array}{c}\text { Blood } \\
\text { pressure } \\
(\mathrm{mm} . \mathrm{Hg})\end{array}$ & $\begin{array}{c}\text { Heart } \\
\text { rate } \\
\text { (beats/min.) }\end{array}$ & $\begin{array}{l}\text { Cardiac } \\
\text { output } \\
\text { (l./min.) }\end{array}$ & $\begin{array}{c}\text { Stroke } \\
\text { volume } \\
\text { (ml.) }\end{array}$ & $\begin{array}{c}\text { Blood } \\
\text { pressure } \\
(\mathrm{mm} . \mathbf{H g})\end{array}$ \\
\hline 1 & $\begin{array}{r}2 \\
5 \\
7 \\
13 \\
18\end{array}$ & $\begin{array}{l}80 \\
76 \\
77 \\
74 \\
74 \\
\end{array}$ & $\begin{array}{l}3 \cdot 70 \\
4 \cdot 42 \\
4 \cdot 25 \\
4 \cdot 17 \\
4 \cdot 64\end{array}$ & $\begin{array}{l}46 \\
58 \\
55 \\
56 \\
63\end{array}$ & $\begin{array}{l}140 / 80 \\
140 / 85 \\
130 / 70 \\
130 / 90 \\
140 / 85\end{array}$ & $\begin{array}{l}76 \\
76 \\
72 \\
68 \\
66\end{array}$ & $\begin{array}{l}3.92 \\
4.75 \\
4.95 \\
5.10 \\
5.50\end{array}$ & $\begin{array}{l}52 \\
63 \\
69 \\
73 \\
83\end{array}$ & $\begin{array}{l}140 / 85 \\
135 / 85 \\
140 / 80 \\
125 / 75 \\
140 / 90\end{array}$ \\
\hline 2 & $\begin{array}{r}3 \\
5 \\
8 \\
16\end{array}$ & $\begin{array}{l}59 \\
62 \\
50 \\
70\end{array}$ & $\begin{array}{l}5 \cdot 20 \\
6 \cdot 55 \\
5 \cdot 00 \\
5 \cdot 89\end{array}$ & $\begin{array}{r}88 \\
106 \\
100 \\
84\end{array}$ & $\begin{array}{l}125 / 80 \\
130 / 80 \\
140 / 90\end{array}$ & $\begin{array}{l}52 \\
58 \\
52 \\
68\end{array}$ & $\begin{array}{l}5 \cdot 68 \\
7 \cdot 27 \\
7 \cdot 17 \\
7 \cdot 06 \\
\end{array}$ & $\begin{array}{l}109 \\
125 \\
138 \\
135\end{array}$ & $\begin{array}{l}130 / 85 \\
130 / 70 \\
150 / 90\end{array}$ \\
\hline 3 & $\begin{array}{r}2 \\
5 \\
8 \\
28\end{array}$ & $\begin{array}{l}76 \\
72 \\
72 \\
76\end{array}$ & $\begin{array}{l}6 \cdot 18 \\
6 \cdot 26 \\
6 \cdot 54 \\
6 \cdot 70\end{array}$ & $\begin{array}{l}81 \\
87 \\
91 \\
88\end{array}$ & $\begin{array}{l}125 / 85 \\
140 / 100 \\
120 / 90 \\
135 / 100\end{array}$ & $\begin{array}{l}\overline{72} \\
80 \\
80\end{array}$ & $\begin{array}{l}-\overline{6 \cdot 39} \\
7 \cdot 84 \\
8 \cdot 11\end{array}$ & $\begin{array}{r}\overline{89} \\
98 \\
101\end{array}$ & $\begin{array}{c}\overline{140 / 95} \\
135 / 90 \\
135 / 95\end{array}$ \\
\hline 4 & $\begin{array}{r}2 \\
4 \\
7 \\
12 \\
17 \\
\end{array}$ & $\begin{array}{l}76 \\
75 \\
76 \\
70 \\
73 \\
\end{array}$ & $\begin{array}{l}6 \cdot 80 \\
7 \cdot 22 \\
6 \cdot 04 \\
5 \cdot 10 \\
5 \cdot 29\end{array}$ & $\begin{array}{l}89 \\
96 \\
80 \\
73 \\
71 \\
\end{array}$ & $\begin{array}{l}130 / 85 \\
140 / 100 \\
135 / 100 \\
120 / 90 \\
115 / 90\end{array}$ & $\begin{array}{l}75 \\
80 \\
80 \\
73 \\
72 \\
\end{array}$ & $\begin{array}{l}7 \cdot 95 \\
9 \cdot 67 \\
7 \cdot 73 \\
6 \cdot 88 \\
6 \cdot 86\end{array}$ & $\begin{array}{r}106 \\
121 \\
97 \\
94 \\
95\end{array}$ & $\begin{array}{l}120 / 80 \\
130 / 90 \\
135 / 105 \\
120 / 90 \\
115 / 95\end{array}$ \\
\hline 5 & $\begin{array}{r}\text { D1 } \\
\text { D4 } \\
2 \\
4 \\
8 \\
11 \\
17 \\
\end{array}$ & $\begin{array}{r}80 \\
100 \\
72 \\
72 \\
70 \\
62 \\
60 \\
\end{array}$ & $\begin{array}{l}5 \cdot 60 \\
4 \cdot 70 \\
4 \cdot 80 \\
5 \cdot 58 \\
6 \cdot 65 \\
5 \cdot 18 \\
5 \cdot 75 \\
\end{array}$ & $\begin{array}{l}70 \\
47 \\
67 \\
78 \\
95 \\
84 \\
96 \\
\end{array}$ & $\begin{array}{l}130 / 100 \\
110 / 100 \\
140 / 90 \\
150 / 120 \\
160 / 130 \\
190 / 160 \\
160 / 125 \\
\end{array}$ & $\begin{array}{l}- \\
84 \\
75 \\
70 \\
60 \\
60\end{array}$ & $\begin{array}{l}- \\
\overline{5 \cdot 69} \\
6 \cdot 22 \\
8 \cdot 44 \\
6 \cdot 16 \\
6 \cdot 50\end{array}$ & $\begin{array}{r}- \\
68 \\
83 \\
121 \\
103 \\
108\end{array}$ & $\begin{array}{l}\overline{-} \\
135 / 90 \\
150 / 125 \\
170 / 140 \\
170 / 140 \\
150 / 120\end{array}$ \\
\hline 6 & $\begin{array}{r}\text { D1 } \\
\text { D3 } \\
2 \\
4 \\
7 \\
12 \\
17 \\
\end{array}$ & $\begin{array}{l}62 \\
83 \\
72 \\
84 \\
82 \\
76 \\
82 \\
\end{array}$ & $\begin{array}{l}3 \cdot 83 \\
4.96 \\
5 \cdot 89 \\
6 \cdot 53 \\
7.54 \\
5 \cdot 68 \\
6.00 \\
\end{array}$ & $\begin{array}{l}55 \\
59 \\
82 \\
78 \\
92 \\
75 \\
73 \\
\end{array}$ & $\begin{array}{c}80 / 55 \\
100 / 75 \\
95 / 65 \\
120 / 90 \\
135 / 105 \\
135 / 105 \\
140 / 100 \\
\end{array}$ & $\begin{array}{l}- \\
66 \\
84 \\
82 \\
76 \\
80\end{array}$ & $\begin{array}{c}- \\
- \\
7 \cdot 07 \\
7 \cdot 90 \\
9 \cdot 85 \\
7 \cdot 84 \\
7 \cdot 38 \\
\end{array}$ & $\begin{array}{r}- \\
107 \\
94 \\
123 \\
103 \\
92\end{array}$ & $\begin{array}{l}\overline{-} \\
100 / 75 \\
120 / 70 \\
125 / 95 \\
130 / 100 \\
135 / 105\end{array}$ \\
\hline 7 & $\begin{array}{r}\text { D1 } \\
\text { D2 } \\
\text { D5 } \\
3 \\
6 \\
11 \\
20 \\
26 \\
\end{array}$ & $\begin{array}{r}120 \\
120 \\
120 \\
110 \\
66 \\
84 \\
84 \\
88 \\
\end{array}$ & $\begin{array}{l}3 \cdot 00 \\
3 \cdot 60 \\
2 \cdot 40 \\
4 \cdot 14 \\
4 \cdot 90 \\
3 \cdot 67 \\
4 \cdot 12 \\
3 \cdot 10\end{array}$ & $\begin{array}{l}25 \\
30 \\
20 \\
38 \\
74 \\
44 \\
49 \\
35\end{array}$ & $\begin{array}{l}125 / 90 \\
120 / 90 \\
120 / 80 \\
115 / 80 \\
130 / 105 \\
125 / 80 \\
115 / 85\end{array}$ & $\begin{array}{l}= \\
\bar{Z} \\
\overline{64} \\
90 \\
90 \\
-\end{array}$ & $\begin{array}{l}= \\
= \\
5 \cdot 14 \\
3 \cdot 60 \\
4 \cdot 47 \\
=\end{array}$ & $\begin{array}{l}\text { 二 } \\
\bar{Z} \\
80 \\
40 \\
50 \\
-\end{array}$ & $\begin{array}{c}\bar{Z} \\
\bar{Z} \\
120 / 75 \\
135 / 90 \\
120 / 85 \\
-\end{array}$ \\
\hline 8 & $\begin{array}{r}\mathrm{D} 3 \\
2 \\
5 \\
8 \\
12 \\
17 \\
\end{array}$ & $\begin{array}{l}59 \\
64 \\
54 \\
55 \\
56 \\
52 \\
\end{array}$ & $\begin{array}{l}2 \cdot 92 \\
5 \cdot 13 \\
4 \cdot 23 \\
4 \cdot 42 \\
4 \cdot 99 \\
5 \cdot 44 \\
\end{array}$ & $\begin{array}{r}49 \\
80 \\
78 \\
82 \\
89 \\
105 \\
\end{array}$ & $\begin{array}{l}120 / 80 \\
150 / 90 \\
135 / 90 \\
145 / 95 \\
170 / 90 \\
140 / 85 \\
\end{array}$ & $\begin{array}{l}\overline{65} \\
57 \\
56 \\
51\end{array}$ & $\begin{array}{l}\overline{6 \cdot 78} \\
5 \cdot 80 \\
\overline{6 \cdot 34} \\
7 \cdot 02\end{array}$ & $\begin{array}{r}1 \overline{104} \\
102 \\
1 \overline{13} \\
138 \\
\end{array}$ & $\begin{array}{l}15 \overline{0} / 85 \\
130 / 90 \\
18 \overline{0} \\
140 / 90 \\
\end{array}$ \\
\hline 9 & $\begin{array}{r}2 \\
8 \\
13 \\
19 \\
\end{array}$ & $\begin{array}{l}80 \\
72 \\
70 \\
74 \\
\end{array}$ & $\begin{array}{l}5 \cdot 60 \\
6 \cdot 56 \\
6 \cdot 74 \\
6 \cdot 86 \\
\end{array}$ & $\begin{array}{l}70 \\
91 \\
96 \\
93 \\
\end{array}$ & $\begin{array}{l}120 / 80 \\
165 / 120 \\
150 / 100 \\
135 / 100 \\
\end{array}$ & $\begin{array}{l}76 \\
70 \\
76 \\
80 \\
\end{array}$ & $\begin{array}{l}5 \cdot 77 \\
7 \cdot 41 \\
7 \cdot 68 \\
8 \cdot 80 \\
\end{array}$ & $\begin{array}{r}76 \\
106 \\
101 \\
110 \\
\end{array}$ & $\begin{array}{l}180 / 110 \\
135 / 90 \\
130 / 100 \\
120 / 85\end{array}$ \\
\hline 10 & $\begin{array}{r}\text { D2 } \\
2 \\
4 \\
8 \\
16 \\
22\end{array}$ & $\begin{array}{l}68 \\
74 \\
72 \\
74 \\
60 \\
76\end{array}$ & $\begin{array}{l}4 \cdot 01 \\
5 \cdot 91 \\
4 \cdot 83 \\
4 \cdot 82 \\
4 \cdot 18 \\
5 \cdot 14\end{array}$ & $\begin{array}{l}59 \\
80 \\
67 \\
65 \\
70 \\
68 \\
\end{array}$ & $\begin{array}{r}90 / 55 \\
110 / 75 \\
135 / 90 \\
130 / 90 \\
125 / 95 \\
120 / 95 \\
\end{array}$ & $\begin{array}{l}\overline{70} \\
73 \\
72 \\
62 \\
68\end{array}$ & $\begin{array}{l}\overline{9 \cdot 10} \\
6 \cdot 52 \\
6 \cdot 89 \\
5 \cdot 22 \\
6 \cdot 32 \\
\end{array}$ & $\begin{array}{r}\overline{130} \\
89 \\
96 \\
84 \\
93 \\
\end{array}$ & $\begin{array}{c}\overline{-} \\
105 / 75 \\
115 / 75 \\
125 / 85 \\
125 / 95 \\
115 / 80\end{array}$ \\
\hline 11 & $\begin{array}{r}\mathrm{D} 1 \\
\mathrm{D} 2 \\
2 \\
12 \\
16\end{array}$ & $\begin{array}{r}116 \\
114 \\
80 \\
90 \\
69 \\
\end{array}$ & $\begin{array}{l}4 \cdot 02 \\
3 \cdot 74 \\
2 \cdot 97 \\
4 \cdot 03 \\
4 \cdot 29 \\
\end{array}$ & $\begin{array}{l}35 \\
33 \\
37 \\
45 \\
62 \\
\end{array}$ & $\begin{array}{r}85 / 50 \\
100 / 70 \\
115 / 90 \\
110 / 85 \\
130 / 85 \\
\end{array}$ & $\begin{array}{l}- \\
92 \\
94 \\
75\end{array}$ & $\begin{array}{l}\text { 二 } \\
3 \cdot 30 \\
2 \cdot 91 \\
4 \cdot 33\end{array}$ & $\begin{array}{l}\bar{Z} \\
36 \\
30 \\
57\end{array}$ & $\begin{array}{c}- \\
115 / 95 \\
110 / 85 \\
130 / 90\end{array}$ \\
\hline 12 & $\begin{array}{r}2 \\
4 \\
7 \\
11 \\
16\end{array}$ & $\begin{array}{l}84 \\
64 \\
70 \\
66 \\
62\end{array}$ & $\begin{array}{l}4 \cdot 33 \\
4 \cdot 11 \\
3 \cdot 45 \\
3 \cdot 91 \\
3 \cdot 90\end{array}$ & $\begin{array}{l}52 \\
64 \\
49 \\
59 \\
63\end{array}$ & $\begin{array}{l}105 / 75 \\
130 / 100 \\
125 / 100 \\
150 / 120 \\
120 / 95\end{array}$ & $\begin{array}{l}83 \\
64 \\
71 \\
68 \\
66\end{array}$ & $\begin{array}{l}5 \cdot 28 \\
5 \cdot 01 \\
4 \cdot 45 \\
4 \cdot 85 \\
5 \cdot 38\end{array}$ & $\begin{array}{l}64 \\
78 \\
63 \\
71 \\
82\end{array}$ & $\begin{array}{l}105 / 80 \\
120 / 90 \\
125 / 100 \\
130 / 105 \\
120 / 95\end{array}$ \\
\hline
\end{tabular}

Table continued on next page. 
TABLE-continued

\begin{tabular}{|c|c|c|c|c|c|c|c|c|c|}
\hline \multirow[b]{2}{*}{$\begin{array}{l}\text { Patient } \\
\text { No. }\end{array}$} & \multirow[b]{2}{*}{$\begin{array}{c}\text { Days } \\
\text { (D1-D7) or } \\
\text { weeks (2-28) } \\
\text { after } \\
\text { infarction }\end{array}$} & \multicolumn{4}{|c|}{ Position 1} & \multicolumn{4}{|c|}{ Position 2} \\
\hline & & $\begin{array}{c}\text { Heart } \\
\text { rate } \\
\text { (beats/min.) }\end{array}$ & $\begin{array}{l}\text { Cardiac } \\
\text { output } \\
\text { (1./min.) }\end{array}$ & $\begin{array}{c}\text { Stroke } \\
\text { volume } \\
(\text { ml. })\end{array}$ & $\begin{array}{c}\text { Blood } \\
\text { pressure } \\
\text { (mm. Hg) }\end{array}$ & $\begin{array}{c}\text { Heart } \\
\text { rate } \\
\text { (beats/min.) }\end{array}$ & $\begin{array}{l}\text { Cardiac } \\
\text { output } \\
\text { (1./min.) }\end{array}$ & $\begin{array}{c}\text { Stroke } \\
\text { volume } \\
(\text { ml. })\end{array}$ & $\begin{array}{c}\text { Blood } \\
\text { pressure } \\
(\mathrm{mm} . \mathrm{Hg})\end{array}$ \\
\hline 13 & $\begin{array}{r}2 \\
4 \\
8 \\
17\end{array}$ & $\begin{array}{l}84 \\
63 \\
72 \\
74\end{array}$ & $\begin{array}{l}5 \cdot 19 \\
5 \cdot 66 \\
5.76 \\
6 \cdot 41\end{array}$ & $\begin{array}{l}62 \\
90 \\
80 \\
87\end{array}$ & $\begin{array}{l}165 / 105 \\
160 / 100 \\
190 / 110 \\
160 / 110\end{array}$ & $\begin{array}{l}76 \\
64 \\
68 \\
74\end{array}$ & $\begin{array}{l}5.48 \\
7.08 \\
7.89 \\
7.38\end{array}$ & $\begin{array}{r}72 \\
111 \\
116 \\
100\end{array}$ & $\begin{array}{l}165 / 110 \\
170 / 100 \\
200 / 120 \\
170 / 110\end{array}$ \\
\hline 14 & $\begin{array}{r}\mathrm{D} 3 \\
\mathrm{D} 5 \\
3 \\
7 \\
12 \\
15\end{array}$ & $\begin{array}{l}63 \\
55 \\
74 \\
74 \\
68 \\
66\end{array}$ & $\begin{array}{l}5 \cdot 46 \\
4 \cdot 18 \\
5 \cdot 68 \\
4.72 \\
5 \cdot 06 \\
4 \cdot 50\end{array}$ & $\begin{array}{l}87 \\
76 \\
77 \\
64 \\
74 \\
68\end{array}$ & $\begin{array}{l}100 / 80 \\
1 \overline{15 / 90} \\
120 / 90 \\
130 / 100 \\
130 / 90\end{array}$ & $\begin{array}{l}7 \\
74 \\
70 \\
68 \\
67\end{array}$ & $\begin{array}{l}\overline{-} \\
6.93 \\
5.29 \\
7.53 \\
6.48\end{array}$ & $\begin{array}{r}\overline{-} \\
94 \\
76 \\
110 \\
97\end{array}$ & $\begin{array}{l}\overline{\overline{1}} \\
120 / 90 \\
125 / 95 \\
130 / 100 \\
120 / 85\end{array}$ \\
\hline 15 & $\begin{array}{r}\text { D3 } \\
2 \\
4 \\
10 \\
15\end{array}$ & $\begin{array}{l}84 \\
78 \\
80 \\
74 \\
70\end{array}$ & $\begin{array}{l}4 \cdot 12 \\
4 \cdot 20 \\
4 \cdot 54 \\
4 \cdot 15 \\
3 \cdot 64\end{array}$ & $\begin{array}{l}49 \\
54 \\
56 \\
56 \\
52\end{array}$ & $\begin{array}{l}135 / 85 \\
155 / 95 \\
145 / 90 \\
160 / 100 \\
155 / 100\end{array}$ & $\begin{array}{l}78 \\
80 \\
76 \\
72\end{array}$ & $\begin{array}{l}4 \cdot \overline{4} \\
4 \cdot 86 \\
5 \cdot 10 \\
4 \cdot 15\end{array}$ & $\begin{array}{l}\overline{58} \\
61 \\
67 \\
58\end{array}$ & $\begin{array}{l}14 \overline{140 / 90} \\
140 / 85 \\
150 / 100 \\
160 / 90\end{array}$ \\
\hline 16 & $\begin{array}{r}\mathrm{D} 2 \\
2 \\
4 \\
7 \\
11 \\
16\end{array}$ & $\begin{array}{l}72 \\
70 \\
70 \\
72 \\
66 \\
66\end{array}$ & $\begin{array}{l}4 \cdot 56 \\
5 \cdot 08 \\
4 \cdot 52 \\
3.99 \\
4 \cdot 28 \\
3 \cdot 49\end{array}$ & $\begin{array}{l}63 \\
73 \\
65 \\
55 \\
65 \\
53\end{array}$ & $\begin{array}{l}240 / 120 \\
190 / 105 \\
190 / 110 \\
200 / 110 \\
160 / 110 \\
180 / 105\end{array}$ & $\begin{array}{l}7 \overline{72} \\
70 \\
82 \\
68 \\
70\end{array}$ & $\begin{array}{l}5 \cdot-89 \\
4 \cdot 88 \\
5 \cdot 47 \\
5 \cdot 18 \\
4 \cdot 30\end{array}$ & $\begin{array}{l}82 \\
70 \\
67 \\
76 \\
61\end{array}$ & $\begin{array}{l}19 \overline{1} \overline{/ 105} \\
180 / 110 \\
165 / 120 \\
180 / 105\end{array}$ \\
\hline 17 & $\begin{array}{r}\mathrm{D} 2 \\
\mathrm{D} 5 \\
2 \\
4 \\
7 \\
10 \\
16\end{array}$ & $\begin{array}{l}76 \\
96 \\
80 \\
74 \\
78 \\
72 \\
68\end{array}$ & $\begin{array}{l}4 \cdot 35 \\
4 \cdot 45 \\
4 \cdot 30 \\
4 \cdot 49 \\
5 \cdot 02 \\
4 \cdot 73 \\
4 \cdot 57\end{array}$ & $\begin{array}{l}57 \\
46 \\
54 \\
61 \\
64 \\
66 \\
70\end{array}$ & $\begin{array}{l}130 / 80 \\
130 / 95 \\
160 / 115 \\
160 / 15 \\
150 / 110 \\
160 / 110\end{array}$ & $\begin{array}{l}7 \overline{7} \\
76 \\
72 \\
78 \\
70 \\
64\end{array}$ & $\begin{array}{l}\overline{7} \\
5.89 \\
5.52 \\
5.97 \\
5.58 \\
5.00\end{array}$ & $\begin{array}{l}\overline{78} \\
78 \\
77 \\
77 \\
80\end{array}$ & $\begin{array}{c}\overline{\overline{1}} \\
130 / 90 \\
140 / 100 \\
165 / 110 \\
150 / 105 \\
155 / 105\end{array}$ \\
\hline 18 & $\begin{array}{r}2 \\
4 \\
7 \\
11 \\
16 \\
\end{array}$ & $\begin{array}{l}66 \\
62 \\
68 \\
67 \\
76 \\
\end{array}$ & $\begin{array}{l}5 \cdot 76 \\
5.95 \\
5 \cdot 60 \\
6 \cdot 28 \\
5 \cdot 2 \\
\end{array}$ & $\begin{array}{l}87 \\
96 \\
82 \\
94 \\
68 \\
\end{array}$ & $\begin{array}{l}165 / 110 \\
160 / 100 \\
150 / 100 \\
155 / 110 \\
150 / 110 \\
\end{array}$ & $\begin{array}{l}68 \\
70 \\
73 \\
69 \\
76 \\
\end{array}$ & $\begin{array}{l}6.09 \\
7 \cdot 02 \\
6.55 \\
6 \cdot 47 \\
6 \cdot 2\end{array}$ & $\begin{array}{r}90 \\
100 \\
90 \\
94 \\
82 \\
\end{array}$ & $\begin{array}{l}160 / 105 \\
160 / 110 \\
150 / 105 \\
150 / 100 \\
150 / 110\end{array}$ \\
\hline 19 & $\begin{array}{r}\text { D2 } \\
\text { D5 } \\
2 \\
4 \\
7 \\
9 \\
16\end{array}$ & $\begin{array}{r}114 \\
102 \\
96 \\
86 \\
92 \\
104 \\
95\end{array}$ & $\begin{array}{l}5 \cdot 62 \\
4 \cdot 71 \\
4 \cdot 76 \\
4 \cdot 85 \\
4 \cdot 08 \\
4 \cdot 26 \\
4 \cdot 75\end{array}$ & $\begin{array}{l}49 \\
46 \\
50 \\
56 \\
44 \\
41 \\
50\end{array}$ & $\begin{array}{l}150 / 100 \\
130 / 80 \\
135 / 95 \\
130 / 90 \\
150 / 110 \\
185 / 115\end{array}$ & $\begin{array}{r}\overline{-} \\
90 \\
84 \\
98 \\
109 \\
98\end{array}$ & $\begin{array}{l}\overline{-} \\
5 \cdot 28 \\
6.45 \\
5.87 \\
6.43 \\
5.94\end{array}$ & $\begin{array}{l} \\
59 \\
77 \\
60 \\
59 \\
60\end{array}$ & $\begin{array}{c}\overline{\bar{c}} \\
130 / 80 \\
135 / 85 \\
130 / 90 \\
165 / 105 \\
180 / 105\end{array}$ \\
\hline 20 & $\begin{array}{r}\mathrm{D} 3 \\
\mathrm{D} 7 \\
2 \\
4 \\
7 \\
11 \\
16 \\
\end{array}$ & $\begin{array}{l}84 \\
74 \\
70 \\
70 \\
80 \\
90 \\
84 \\
\end{array}$ & $\begin{array}{l}5 \cdot 41 \\
5.79 \\
6.76 \\
5.25 \\
5.62 \\
7.67 \\
4.85 \\
\end{array}$ & $\begin{array}{l}64 \\
78 \\
97 \\
75 \\
70 \\
85 \\
57 \\
\end{array}$ & $\begin{array}{l}140 / 80 \\
190 / 105 \\
200 / 10 \\
175 / 100 \\
185 / 115 \\
200 / 110 \\
\end{array}$ & $\begin{array}{l}\overline{7} \\
72 \\
68 \\
79 \\
96 \\
89 \\
\end{array}$ & $\begin{array}{c}7 \\
7.71 \\
6.51 \\
7.53 \\
10.4 \\
6.02 \\
\end{array}$ & $\begin{array}{r}\overline{-} \\
107 \\
96 \\
95 \\
107 \\
71\end{array}$ & $\begin{array}{c}\overline{\overline{1}} \\
175 / 95 \\
170 / 100 \\
180 / 100 \\
180 / 105 \\
188 / 110\end{array}$ \\
\hline 21 & $\begin{array}{r}\text { D2 } \\
\text { D5 } \\
4 \\
7 \\
11 \\
16 \\
\end{array}$ & $\begin{array}{l}86 \\
78 \\
84 \\
87 \\
87 \\
88 \\
\end{array}$ & $\begin{array}{l}4 \cdot 30 \\
4 \cdot 23 \\
3 \cdot 86 \\
3 \cdot 73 \\
5 \cdot 05 \\
5 \cdot 19 \\
\end{array}$ & $\begin{array}{l}50 \\
54 \\
47 \\
43 \\
58 \\
59 \\
\end{array}$ & $\begin{array}{r}90 / 60 \\
95 / 60 \\
110 / 80 \\
140,95 \\
130 / 90 \\
135 / 90 \\
\end{array}$ & $\begin{array}{l}\overline{7} \\
88 \\
92 \\
87 \\
86\end{array}$ & $\begin{array}{l}\overline{-} \\
5 \cdot 63 \\
4 \cdot 02 \\
5 \cdot 89 \\
5 \cdot 97\end{array}$ & $\begin{array}{l}\overline{7} \\
64 \\
44 \\
68 \\
69\end{array}$ & $\begin{array}{c}- \\
110 / 85 \\
120 / 90 \\
130 / 85 \\
130 / 85\end{array}$ \\
\hline 22 & $\begin{array}{r}\text { D3 } \\
\text { D5 } \\
2 \\
4 \\
7 \\
11 \\
16\end{array}$ & $\begin{array}{r}102 \\
95 \\
86 \\
97 \\
89 \\
80 \\
81\end{array}$ & $\begin{array}{l}6 \cdot 74 \\
5 \cdot 35 \\
4 \cdot 68 \\
4 \cdot 66 \\
4.40 \\
4 \cdot 21 \\
3.99\end{array}$ & $\begin{array}{l}66 \\
56 \\
54 \\
48 \\
49 \\
53 \\
49\end{array}$ & $\begin{array}{l}170 / 105 \\
140 / 95 \\
130 / 100 \\
165 / 115 \\
145 / 110\end{array}$ & $\begin{array}{l}\bar{Z} \\
100 \\
89 \\
80 \\
73\end{array}$ & $\begin{array}{c}\bar{Z} \\
\overline{5} \\
\begin{array}{c}5 \cdot 26 \\
4.84 \\
4 \cdot 48\end{array}\end{array}$ & $\begin{array}{l} \\
\overline{53} \\
54 \\
56 \\
64\end{array}$ & $\begin{array}{c}\overline{\bar{Z}} \\
130 / 100 \\
\overline{165 / 105} \\
145 / 105\end{array}$ \\
\hline
\end{tabular}

showed a fall. In patients 4 and 18 , the stroke volume remained above $60 \mathrm{ml}$. In patients 16 and 20 stroke volume was below $60 \mathrm{ml}$. at the final follow-up study, possibly in relation to a further small myocardial infarction in the thirteenth week in patient 16 , and hypertension rising to $200 / 110$ $\mathrm{mm} . \mathrm{Hg}$ in patient 20 . The mean stroke volume in the 13 patients at the first study in early convalescence was $80 \mathrm{ml}$. and at the end of the follow-up period it was $78 \mathrm{ml}$. 


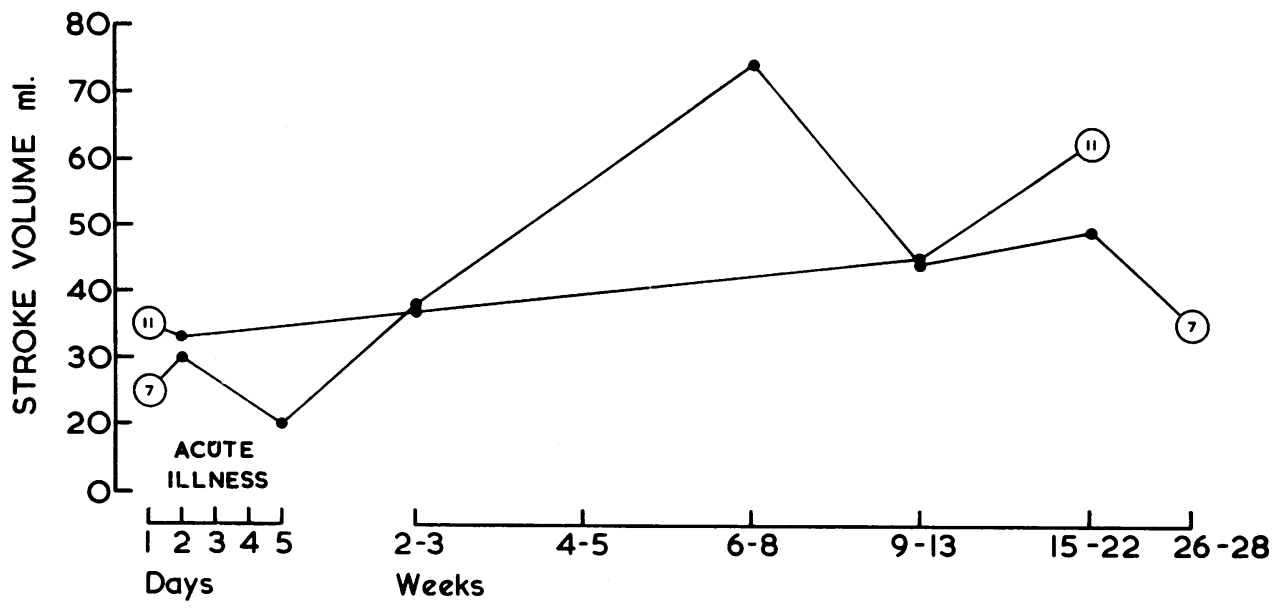

TIME OF INVESTIGATION

FIG. 3.-Changes in stroke volume occurring in those patients with stroke volume below $40 \mathrm{ml}$. at the first study in the early convalescent period (Group III).

Seven patients $(1,12,15,17,19,21,22)$ had a stroke volume between 40 and $60 \mathrm{ml}$. at the first investigation of the convalescent period. Of these, $4(1,12,17,21)$ showed an increase during the follow-up period and $3(15,19,22)$ showed no change. The average stroke volume in the 7 patients at the first study in early convalescence was $52 \mathrm{ml}$. and at the end of the follow-up period $58 \mathrm{ml}$.

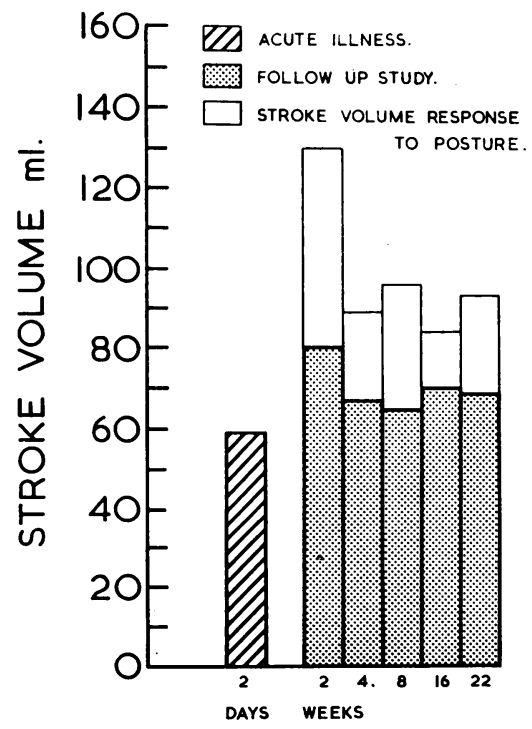

Fig. 4. Patient 10: a representative example of a good stroke volume response to the standard postural change.
Two patients $(7,11)$ had a stroke volume between 20 and $40 \mathrm{ml}$. (38 and $37 \mathrm{ml}$., respectively) when studied during the second week. In 7 a period of temporary clinical improvement following treatment with digitalis and diuretics was associated with an increase of stroke volume from 38 to $74 \mathrm{ml}$. Subsequent deterioration with a fall in stroke volume to $35 \mathrm{ml}$. occurred after discharge from hospital. In patient 11 the stroke volume at the end of the follow-up period was $62 \mathrm{ml}$.

(3) Heart Rate: Acute phase-early convalescence. Heart rate in the acute phase (14 patients) ranged from 55 to $120 / \mathrm{min}$. Six patients $(5,7,11,17$, $19,22)$ had heart rates above $85 / \mathrm{min}$., five $(6,15$, $16,20,21$ ) between 70 and $85 / \mathrm{min}$., and three $(8,10,14)$ between 55 and $70 / \mathrm{min}$. Within the time between the acute illness and the first investigation of the convalescent period there was a general tendency for high and low heart rates to revert to the normal range. Patients with heart rates in the range $70-85 / \mathrm{min}$. during the acute illness showed little change.

Heart rates at the time of the first study of the convalescent period were within the range 70-85/ min. in all patients except six $(2,7,8,18,19,22)$. Of these six patients, three $(2,8,18)$ had rates below $70 / \mathrm{min}$. and three $(7,19,22)$ above $85 / \mathrm{min}$. Patient 7 had a stroke volume below $40 \mathrm{ml}$. and other evidence of circulatory failure. Patient 19 had a stroke volume of $50 \mathrm{ml}$. and subsequent to the follow-up period developed congestive cardiac failure. Patient 22 had a stroke volume of $54 \mathrm{ml}$. 
Patient I
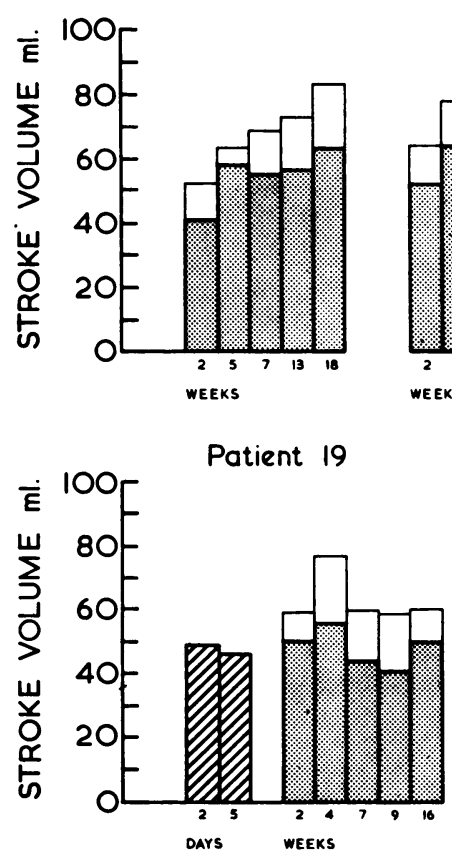

Patient 12

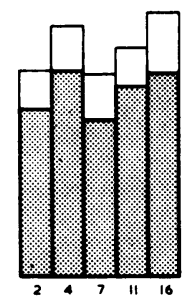

WEEKS
Patient 15

Z ACUTE ILLNESS.

FOLLOW UP STUDY STROKE VOUUME RESPONSE to POSTURE.

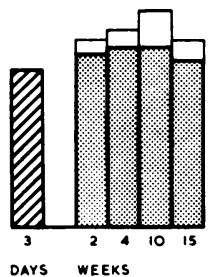

Patient 21

Patient 17
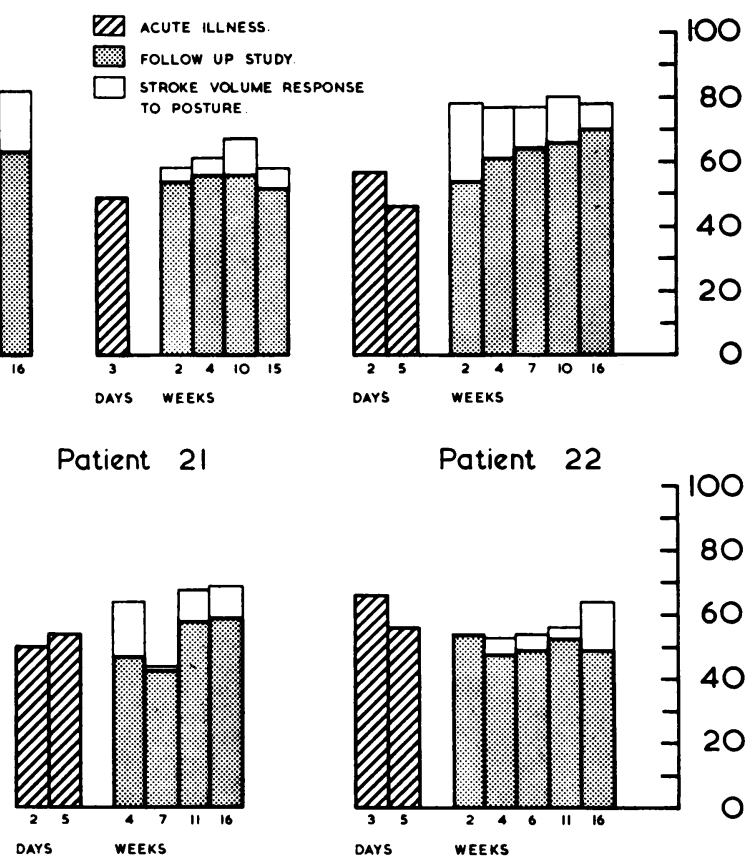

DAYS WEEKS

Fig. 5.-Changes in stroke volume with time and the response to the standard postural change in those patients with stroke volume between 40 and $60 \mathrm{ml}$. at the first study in the early convalescent period.

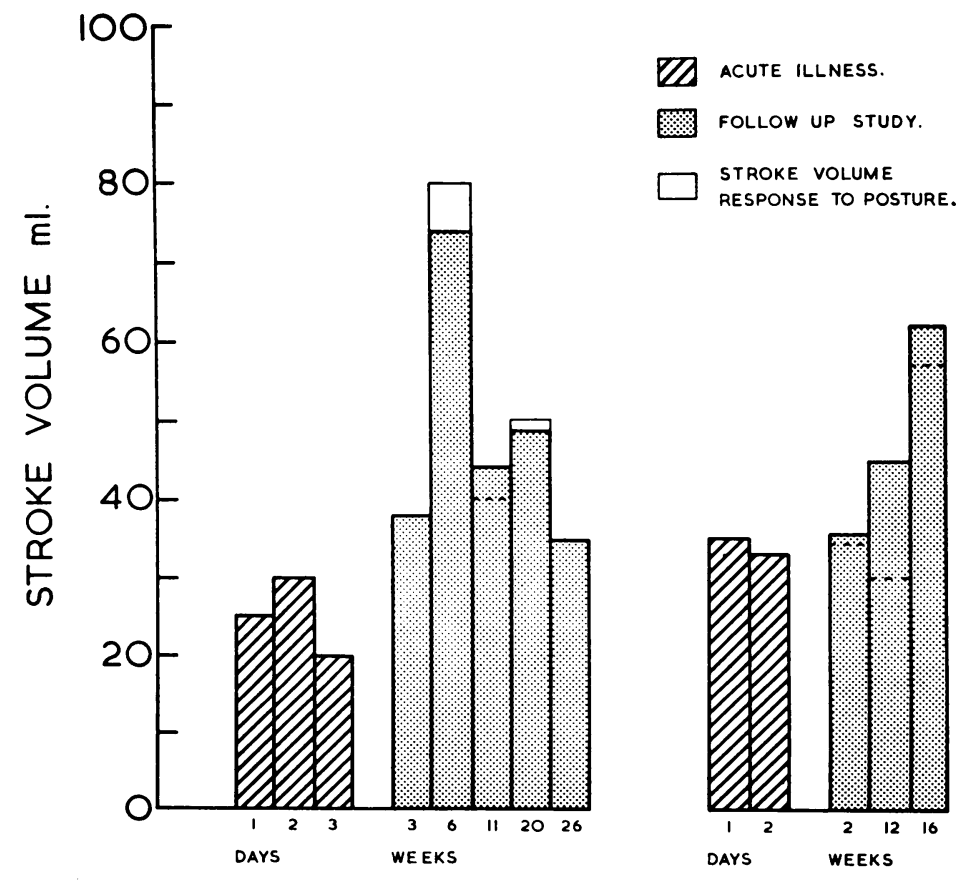

Fig. 6. Changes in stroke volume with time and the response to the standard postural change in those patients with stroke volume of less than $40 \mathrm{ml}$. at the first study in the early convalescent period. Interrupted lines represent a fall in stroke volume. On the left, patient 7; on the right; patient 11. 
Early convalescence-end of follow-up. During the period of follow-up from the second week the 16 patients with heart rate in the range $70-85 / \mathrm{min}$. at the beginning of convalescence behaved as follows. Eight $(1,3,4,6,9,10,16,21)$ showed no significant change in heart rate; seven $(5,11,12$, $13,14,15,17)$ showed a fall, most conspicuous in 11 and 12. One patient (20) showed a progressive increase.

Of the three patients $(7,19,22)$ with heart rate above $85 / \mathrm{min}$. in early convalescence, one (7) showed an initial fall to $66 / \mathrm{min}$., subsequently rising again to $88 / \mathrm{min}$. One (19) remained in the range $85-105 / \mathrm{min}$., and one (22) showed a progressive fall to $80 / \mathrm{min}$.

Of the three patients with heart rates below $70 /$ min. at the beginning of convalescence, two $(2,18)$ subsequently increased to $70 / \mathrm{min}$. or above and one (8) remained low (about $55 / \mathrm{min}$.).

(4) Blood Pressure. In all except five patients $(1,2,3,4,7)$ the blood pressure increased during the period of study. In some $(9,15,17,18,19,20)$ hypertension appeared to varying degrees. Four patients $(5,13,16,22)$ with known previous hypertension either maintained or ultimately reached high levels of arterial pressure. Patient 13 was treated with bethanidine throughout the study and patient 22 was given chlorothiazide for control of arterial pressure between the studies of the eleventh and sixteenth weeks.

\section{Circulatory Response to a STANDARd Postural Change}

The changes in cardiac output, heart rate, stroke volume, and blood pressure, which resulted from the standard postural test conducted at different stages in the follow-up period, are given in the Table. Patients were placed in three groups on a basis of the stroke volume found at the first investigation of the follow-up period (second week in most patients). Group I consists of patients with stroke volume greater than $60 \mathrm{ml}$. in the $20^{\circ}$ feet down position; Group 2 consists of those with stroke volume between 40 and $60 \mathrm{ml}$., and Group 3 those with stroke volume less than $40 \mathrm{ml}$.

Group I. Patients 2, 3, 4, 5, 6, 8, 9, 10, 13, 14, 16,18 , and 20 were placed in this group. The average stroke volume of these patients at all investigations conducted between the second week and the end of the follow-up period was $80.8 \mathrm{ml}$. The average stroke volume in the second position of the posture test was $99.0 \mathrm{ml}$. The average percentage increase of stroke volume was 22.5 per cent. The Table shows that 8 of the 13 patients within this group responded with a large increase of stroke volume following the change in posture at all investigations during the period of follow-up. An example of a typical response is shown in Fig. 4. Two patients $(3,5)$ in whom stroke volume was progressively increasing during the period of follow-up showed a progressively greater stroke volume response to posture. Patient 9 showed only a small stroke volume response at the beginning of convalescence but a greater response at the final study. Patient 20 achieved a good stroke volume response between the fourth and eleventh weeks but this was less at the final study: at the 16th week arterial pressure had risen to $210 / 110 \mathrm{~mm}$. $\mathrm{Hg}$.

The two patients $(16,18)$ who clearly did not increase stroke volume following the change in posture were also exceptional in that patient $16 \mathrm{had}$ very high levels of arterial pressure at all times and suffered a further small myocardial infarction before the final study; patient 18 had evidence of a large functional aneurysm of the left ventricle though maintaining a good resting stroke volume of more than $80 \mathrm{ml}$.

Group II. Patients 1, 12, 15, 17, 19, 21, and 22 were placed in this group. The average stroke volume of these patients at all investigations conducted between the second week and the end of the follow-up period was $54.4 \mathrm{ml}$. The average stroke volume in the second position of the posture test was $66.3 \mathrm{ml}$. The average percentage increase in stroke volume was 21.9 . Fig. 5 illustrates the progress of each individual patient in terms of stroke volume and postural response. Three $(1,12,17)$ showed increases in stroke volume during the period of study; 2 of these ( 1 and 12) showed improving stroke volume response to posture and a consistent moderately good response, respectively, but 17 showed a diminishing response. Patient 21 showed a moderate stroke volume response to posture except at the seventh week. Patients 15, 19 , and 22 showed little change in stroke volume, but in patient 19 the stroke volume response to posture was greater as compared with patients 15 and 22 in whom the response was poor until 16 weeks after cardiac infarction.

Group III. Patients 7 and 11 were placed in this group (Fig. 6). The average stroke volume of these two at all investigations conducted between the second week and the end of the follow-up period was $51.8 \mathrm{ml}$. The average stroke volume in the second position of the posture test was $48.8 \mathrm{ml}$. The average percentage change of stroke volume was a fall of 6 . 
In patient 7 a transient clinical improvement was associated with increased stroke volume but thereafter the stroke volume remained low. Stroke volume either showed little change or decreased after change of posture. Patient 11 showed a progressive increase in stroke volume from 37 to $62 \mathrm{ml}$. but stroke volume fell on every occasion after posture change.

\section{CoRRElation OF HamodyNAMIC Measurements with Clinical Features}

The cardiovascular physical signs and radiological features that were present at the time of hæmodynamic investigations were routinely recorded. Particular note was made of auscultatory signs, the presence or absence of a raised jugular venous pressure, radiological evidence of cardiac enlargement, and signs of pulmonary venous hypertension and pulmonary œdema. Clinical features suggesting ventricular aneurysm were also noted. Results are presented with reference to the findings at the first study in the early convalescent period and patients are divided into three groups.

Group I: patients with stroke volume greater than $60 \mathrm{ml}$. at this time.

Group II: patients with stroke volume between 40 and $60 \mathrm{ml}$.

Group III: patients with stroke volume less than $40 \mathrm{ml}$.

\section{Ausculatory Signs}

Group I: Patients 2, 3, 4, 5, 6, 8, 9, 10, 13, 14, 16, 18 , and 20 were placed in this group. Seven of them were studied in the acute phase at which time four $(5,6,14,16)$ had a fourth heart sound, but this was lost by the time of the first convalescent study.

Three $(8,13,18)$ had a fourth heart sound at the first study in early convalescence. In patient 8 this was lost after five weeks, but in patients 13 and 18 it persisted throughout the whole period of study. Patient 13 had systemic hypertension and patient 18 had clinical evidence of a ventricular aneurysm. Patient 16 had a fourth sound in the acute illness, lost it until the seventh week, when it returned, ultimately to persist. This patient had systemic hypertension. Patient 20 developed a fourth sound at the end of the period of study; he also had systemic hypertension.

Group II. Patients 1, 12, 15, 17, 19, 21, and 22 were placed in this group. Five of them were studied during the acute phase. Four $(15,17,19$, 21) had a fourth sound at this time; patient $17 \mathrm{had}$ a third sound also. Patient 22 had a third sound during the acute illness.

At the time of the first study in early convalescence, five $(1,12,16,17,21)$ had a fourth sound which was lost permanently during the period of study in 3 $(1,15,17)$ and temporarily in one (21). In 2 of these 3 patients $(1,17)$, stroke volume was increasing during the period in which the fourth heart sound was lost and in patient 21 stroke volume increased at the time the fourth sound reappeared. In patient 22 , in whom the arterial pressure rose to hypertensive levels during the follow-up period, a fourth sound appeared at the sixteenth week.

Group III. Patients 7 and 11 were placed in this group. Both of them were studied in the acute phase at which time patient 7 had fourth and third heart sounds and 11 a third heart sound.

At the first study of the convalescent period patient 7 had a fourth heart sound which persisted until the 20th week; from the eleventh week a third heart sound was present. General clinical improvement with a greater stroke volume was present during the time in which the third heart sound was absent. Patient 11 had a third heart sound during the whole period of study, at the end of which some small clinical improvement in terms of exercise tolerance and greater improvement in stroke volume was evident.

\section{Raised Fugular Venous Pressure}

Five $(6,7,11,21,22)$ of the 14 patients studied during the acute phase had a jugular venous pressure $5 \mathrm{~cm}$. or more above the sternal angle at that time.

At the time of the first study in the convalescent period only $3(7,11,18)$ had a raised jugular venous pressure. In patient 18, who had a functional cardiac aneurysm, the venous pressure was normal at subsequent studies. Patients 7 and 11 , in whom the venous pressure remained raised to some extent for many weeks, had stroke volumes of less than $\mathbf{4 0}$ $\mathrm{ml}$. at the beginning of the convalescent period and of 35 and $62 \mathrm{ml}$., respectively, at the end of the period of study (Fig. 3).

\section{Heart Size}

At the time of the first study in convalescence 7 patients $(5,7,11,12,13,15,16)$ had radiological evidence of cardiac enlargement. This was particularly noticeable in 7 and 11 in whom the stroke volume was less than $40 \mathrm{ml}$. The stroke volume in the others ranged from 52 to $73 \mathrm{ml}$. Three $(5,13,16)$ suffered from systemic hypertension and in $2(12,15)$ the blood pressure was significantly high at some stage in the follow-up period. 
Radiological and Clinical Evidence of Pulmonary Venous Hypertension and Pulmonary Oedema

At the time of the first study in early convalescence only 2 patients $(7,11)$ had evidence of pulmonary venous hypertension which persisted during the whole of the period of study. Both had other clinical and hæmodynamic evidence of poor cardiac function, with stroke volume of less than $40 \mathrm{ml}$. at the beginning of the follow-up period.

\section{Ventricular Aneurysm}

Four patients $(2,12,13,18)$ had evidence of a functional ventricular aneurysm. All had a pathological præcordial pulsation and paradoxical ventricular movement as shown by screening. Three $(12,13,18)$ had persistent $S-T$ segment elevation in the electrocardiogram. The stroke volume at the beginning of the convalescent period was $88,52,62$, and $87 \mathrm{ml}$., respectively. Three patients $(2,12,13)$ responded to postural change by an increase in stroke volume but patient 18 showed only a very small stroke volume change. Patients 12, 13, and 18 had a persistent fourth heart sound but also had systemic hypertension.

\section{Discussion}

The long-term changes in the circulation which may follow myocardial infarction have not received the attention that has been directed towards the events of the acute illness. It may be that a knowledge of the circulatory changes occurring during the first months after myocardial infarction in relation to the physical signs shown by patients may assist in their clinical management and also aid in the assessment of prognosis with respect to physical activity.

Previous studies (Murphy et al., 1963) have pointed out that survivors from myocardial infarction usually have either a normal cardiac output during the acute illness or a rapid return of low cardiac output to normal levels during convalescence; whereas a persistently low cardiac output carried a poor prognosis for life. Malmcrona and Varnauskas (1964) studied patients during the acute phase of the illness, before they left hospital, and from 3 to 17 months after the infarction, and found no significant differences in cardiac output during these different stages of the disease. Stroke volume improved in the period between the acute phase and leaving hospital, but did not change thereafter.

While most hæmodynamic studies in cardiac infarction have emphasized the importance of cardiac output, it is probable that from the point of view of the ability of the ventricles to contract, stroke volume is a more representative measurement. The results have, therefore, been presented with particular reference to stroke volume. It should be noted that measurements made in the acute phase were made in the horizontal position and subsequent values in the follow-up period were measured in a $20^{\circ}$ feet down position. Corresponding levels for the horizontal position would be approximately 10 per cent greater in most patients with good cardiac function and less different in those with poor cardiac function.

A general appraisal of the values for stroke volume and the changes that took place introduces the difficulty of adopting criteria for normality. Since all strict conventions are open to some criticism, results have been given simply as absolute values, and division of patients into groups with stroke volume above $60 \mathrm{ml}$., between 40 and $60 \mathrm{ml}$., and below $40 \mathrm{ml}$, in the $20^{\circ}$ feet down position, was made arbitrarily for the organization of results. The associated clinical features shown by patients were generally in support of these divisions being, respectively, representative of patients with good, moderately impaired, and poor cardiac function.

The patterns of change in stroke volume showed trends which may be distinct. Nearly all patients who achieved a stroke volume above $60 \mathrm{ml}$. during the follow-up period reached this level by the second week. Further change after the second week in these patients was variable, but in only two patients, in whom hypertension and further myocardial infarction may have been important, did stroke volume subsequently fall below $60 \mathrm{ml}$. This group of patients with the highest stroke volume showed the greatest and most rapid increase in stroke volume during the period between the acute illness and the second week. Their behaviour differed from that of the patients with a lower stroke volume at the second week who also had reduced stroke volume during the acute illness but in whom increase in stroke volume was much slower. In these latter patients, approximately half had stroke volumes less than $60 \mathrm{ml}$. at the end of the period of study. It appears, therefore, that assessment of patients at the second or third week gives a useful indication of prognosis with regard to stroke volume.

There was some relation between the severity of the acute illness in terms of degree of cardiovascular failure and the subsequent behaviour of stroke volume; in general an uncomplicated illness carried a good prognosis and overt cardiovascular failure a poor prognosis with respect to the stroke volume ultimately achieved. The essential reason for the difference in behaviour in stroke volume was not apparent from the study. A simple explanation in terms of bulk of myocardium affected by infarction 
was denied by the levels of serum lactic dehydrogenase and the extent of electrocardiographic changes. There was no apparent relationship to a history of previous myocardial infarction. It was notable, however, that the mean age of the patients with stroke volume above $60 \mathrm{ml}$. in early convalescence was about a decade lower than those in whom it was below $60 \mathrm{ml}$. (52 years; 63 years). Age may therefore be one factor tending to limit cardiac function after myocardial infarction.

The clinical features shown by the patients at the different times of study after the second week were in accord with the results of the hæmodynamic measurements made, and in general reflected the level of stroke volume and degree of ventricular functional disease. Patients with normal stroke volume usually had normal cardiovascular physical signs though a fourth heart sound was present in those patients with systemic hypertension or ventricular aneurysm. In the absence of hypertension or signs of ventricular aneurysm, the presence of a fourth heart sound was a good indication of some reduction in stroke volume though not necessarily of a severe degree. A third heart sound persisted after the second week, however, which indicated much reduced stroke volume and was associated with other clinical features suggesting very poor cardiac function.

Cardiac size was difficult to evaluate between the period of the acute illness and convalescence, since the initial $x$-ray films were taken with patients in the supine position using a ward unit, and, subsequent to the patient's mobilization, films were taken in the standing position. From the second week onward, when the technique was standard, it seemed apparent that cardiac size was normal or possibly only slightly enlarged in most non-hypertensive patients including those with moderately reduced stroke volume. Systemic hypertension was associated with cardiac enlargement in patients with normal stroke volume, but in people with normal blood pressure obvious cardiac enlargement was present only when stroke volume was very low.

The stroke volume response to a standard postural change was studied in all patients, with a view to exploring its use as a test of ventricular function. As had been observed in previous work (Thomas and Shillingford, 1965), there was a general relation between the ability to increase stroke volume and ventricular function, as assessed by the resting stroke volume and clinical criteria, but the test did not seem suitable for evaluating minor changes in cardiac function in individual patients. One patient with a ventricular aneurysm but a normal resting stroke volume failed to respond to the test, and in others the magnitude of the stroke volume response did not show a constant relation to the resting stroke volume. The irregularities may imply a multiplicity of factors involved in the test itself or possibly reflect a variety of types of ventricular dysfunction.

\section{SUMMARY}

Serial hæmodynamic measurements and clinical observations were made at regular intervals during the first four months after acute myocardial infarction in 22 patients. The natural course of changes in cardiac output and stroke volume was found to differ in patients who eventually had good cardiac function as compared with those in whom a cardiac functional defect remained. Increase in stroke volume was most rapid in those patients who returned to a normal stroke volume, and occurred much more slowly in those patients who failed to return to normal. Correlations between hæmodynamic findings and clinical signs were found and are given in detail.

The use of a standard postural change as a test of cardiac function was explored and its place in objective assessment of the circulation was discussed.

The authors wish to thank Mr. Peter Burgess and Miss Sunja Marrington for technical assistance, and Miss Jean Powell who drew the diagrams.

\section{REFERENCES}

Broch, O. J., Humerfelt, S., Haarstad, J., and Myhre, J. R. (1959). Hemodynamic studies in acute myocardial infarction. Amer. Heart f., 57, 522.

Gabe, I., and Shillingford, J. (1961). The photo-electric earpiece technique for recording dye dilution curves. Brit. Heart f., 23, 271.

Gammill, J. F., Applegarth, J. J., Reed, C. E., Fernald, J. D. and Antenucci, A. J. (1955). Hemodynamic changes following acute myocardial infarction using the dye injection method for cardiac output determination. Ann. intern. Med., 43, 100.

Lee, G. de J. (1957). Total and peripheral blood flow in acute myocardial infarction. Brit. Heart f., 19, 117.

Malmcrona, R., and Varnauskas, E. (1964). Hæmodynamics in acute myocardial infarction. Acta med. scand., 175, 1.

Murphy, G. W., Glick, G., Schreiner, B. F., and Yu, P. N. (1963). Cardiac output in acute myocardial infarction. Serial determination by precordial radioisotope dilution curves. Amer. F. Cardiol., 11, 587.

Pritchard, W. H., and Hellerstein, H. K. (1950). Cardiac catheterization following acute myocardial infarction. f. clin. Invest., 29, 839.

Shillingford, J. P., and Thomas, M. (1964). Organization of unit for intensive care and investigation of patients with acute myocardial infarction. Lancet, 2, 1113. 
Smith, W. W., Wikler, N. S., and Fox, A. C. (1954). Hemodynamic studies of patients with myocardial infarction. Circulation, 9, 352.

Thomas, M., Malmcrona, R., and Shillingford, J. (1965a). The accuracy of the photoelectric earpiece technique in the measurement of cardiac output. Brit. Heart f., 27, 805. $\longrightarrow$ - and - (1965b). Hemodynamic changes in patients with acute myocardial infarction. Circulation, $31,811$.

— a standard postural change in ischæmic heart disease. Brit. Heart F., 27, 17. 